\title{
Desenvolvimento de Instrumentais para Avaliação da Estratégia Saúde da Família em Natal
}

\section{Development of Instruments for Assessing the Family Health Strategy in Natal-RN}

\author{
Cynthia de Freitas Melo Lins", ${ }^{*}$, João Carlos Alchieri ${ }^{b}$, João Lins de Araújo Neto ${ }^{c}$ \\ \& Fabrício Augusto de Freitas Melo ${ }^{d}$ \\ ${ }^{a}$ Universidade de Fortaleza, Fortaleza, Ceará, Brasil \\ ${ }^{b}$ Universidade Federal do Rio Grande do Norte, Natal, Rio Grande do Norte, Brasil, \\ ${ }^{c}$ Escola de Saúde Pública do Ceará, Fortaleza, Ceará, Brasil \\ \& Instituto Federal de Educação, Ciência e Tecnologia do Ceará, Fortaleza, Ceará, Brasil
}

\begin{abstract}
Resumo
A Estratégia Saúde da Família (ESF) é a principal porta de entrada dos serviços do Sistema Único de Saúde, necessitando ser constantemente avaliada para seu aperfeiçoamento. Dessa forma, a presente pesquisa correlacional objetivou avaliar a ESF apreendendo as crenças de seus profissionais, criando um novo instrumento, contando com uma amostra probabilística de 475 profissionais. $\mathrm{O}$ novo instrumento com 24 itens, com escala de notas ( 0 a 10$)$, possui três fatores, todos mal avaliados pelas notas dos profissionais: (a) infraestrutura física (alpha=0,84), com 4,37 $( \pm 1,61)$; (b) recursos materiais (alpha=0,85), nota 5,42 $( \pm 1,47)$; e (c) eficiência no atendimento (alpha=0,82), com 3,99 $( \pm 1,35)$. Conclui-se que a nova escala é relevante e que há necessidade de mudanças nos entraves na operacionalização dos serviços na ESF.

Palavras-chave: Avaliação de programas, Estratégia Saúde da Família, profissionais, crenças.
\end{abstract}

\begin{abstract}
Family Health Strategy (FHS) is the main gateway to the Brazilian National Healthcare System services which needs to be constantly evaluated for its improvement. Therefore, this correlational study aimed to evaluate the FHS, understanding the beliefs of the professionals involved, creating a new instrument and counting with a probability sample of 475 professionals. The new instrument composed by 24 items, with grading scale ( 0 to 10$)$, has three factors. All of them were poorly evaluated by scores given by the professionals: (a) physical infrastructure (alpha $=.84)$ got $4.37( \pm$ $1.61)$; (b) material resources (alpha $=.85)$, grade $5.42( \pm 1.47)$; and $(c)$ health care efficiency (alpha $=.82)$ got $3.99( \pm 1.35)$. It is concluded that the new scale is relevant, but there is also the need for changes in the barriers found in the operationalization of the FHS service.

Keywords: Program Evaluation, Family Healthcare Strategy, professionals, beliefs.
\end{abstract}

A presente pesquisa objetivou criar um novo instrumento de medição para a avaliação da Estratégia Saúde da Família (ESF) a partir das crenças de seus profissionais, bem como avalia-la em Natal, Rio Grande do Norte, buscando assim colaborar com a construção de ferramentas eficazes que auxiliem na compreensão sobre as condições e os fatores que influenciam a operacionalização dos serviços de atenção à saúde da comunidade. Desta forma, é possível incentivar a cultura de avaliação de programas, tornando-as mais sistemáticas e viáveis em larga escala, para desta forma fornecer feedback aos seus gestores e comunidade, auxiliando em decisões sobre medidas para o seu aperfeiçoamento.

"Endereço para correspondência: Av. Sargento Herminio, 1415, Apto. 1503, Bloco Violeta, Lado A, Fortaleza, CE, Brasil 60350-550. E-mail: cf.melo@yahoo.com.br, jcalchieri@gmail.com, joaolinsneto@yahoo.com.br e fabricioaugustofm@yahoo.com.br
A Saúde da Família atua no Brasil desde 1994, através do Programa Saúde da Família (PSF), e em 2006 assume nova nomenclatura, Estratégia Saúde da Família, configurando-se como a estratégia estruturante de orientação da Atenção Básica do Sistema Único de Saúde (SUS). Uma estratégia respaldada nos princípios de integralidade e hierarquização, que está vinculada à rede de serviços do SUS e que representa a porta principal de acesso ao sistema, por meio da qual os usuários possuem acesso aos serviços básicos de ações generalistas, podendo, sempre que necessário, serem encaminhados para serviços especializados de maior complexidade, na Atenção Secundária e/ou Atenção Terciária, de forma que seja assegurada a referência (encaminhamento) e contra referência (retorno do encaminhamento) e garantida à atenção integral aos indivíduos e às famílias (Ministério da Saúde, 2004a, 2006).

A atuação da Estratégia Saúde da Família fundamenta-se ainda sobre os ideais de prevenção de doenças e de promoção e recuperação da saúde, através de uma equipe 
multidisciplinar mínima, a Equipe de Saúde da Família (EqSF), composta por um médico, um enfermeiro, um auxiliar de enfermagem (AE), um dentista, um auxiliar de consultório dentário (ACD) e de quatro a seis agentes comunitários de saúde (ACS), que atuam prioritariamente nas Unidades de Saúde da Família (USFs), realizando também visitas domiciliares e escolares, com jornada de trabalho de 40 horas (Ministério da Saúde, 2001, 2006). Contempla-se ainda que em cada USF pode haver mais de uma equipe, sendo o trabalho destas separados, em que cada uma possui seu território de abrangência definido, onde são responsáveis pelo cadastramento e o acompanhamento da população vinculada a esta área: 600 a 1.000 famílias, não devendo ultrapassar o máximo de 4.500 pessoas, sendo a média recomendada de 3.000 habitantes (Ministério da Saúde, 2001, 2004a, 2006). No caso de Natal, o município é dividido em cinco Distritos Sanitários, cada um responsável por gerir as Unidades de Saúde da Família (USFs) existentes em seu território (num total de 37 USFs na cidade), e cada uma dessas é responsável por ruas e residências pré-definidas. A destacar que cada DS possuem índices de qualidade de vida (IQV), construto composto por três dimensões (renda, ambiental e educação), por bairros bastante diferenciados, apresentando-se do melhor para o pior: DS Sul ( 5 bairros com IQV alto e 2 médios), DS Leste (4 altos, 7 médios e 1 baixo), DS Oeste (4 médios e 5 baixos), DS Norte 2 (1 médio e 2 baixos) e DS Norte 1 (4 baixos; Barroso, 2003).

Um modelo que, se não perfeito, ideal, inspirado em países como Cuba e Canadá, mas que tem sido alvo constate de críticas pelos seus usuários, mídia e comunidade acadêmica, pois a realidade de atenção à saúde no Brasil é muito adversa ao modelo teórico idealizado de acesso universal, integral, hierarquizado e em equidade (Albuquerque \& Melo, 2010; Albuquerque, Melo, Sousa Filho, 2011; Melo, 2009).

As avaliação de programas sociais e estratégias governamentais surgem como ferramenta fundamental para auxiliar, através de feedback, nas decisões dos gestores no tocante à implementação, ao processo e aos resultados alcançados pelos seus serviços (Belloni, Magalhães, \& Sousa, 2003), na busca da melhoria dos serviços e mitigando a crença brasileira de que serviços públicos são para pobres e que, portanto, podem ser de baixa qualidade (Albuquerque, 2002), tentando, assim, instigar a população, como cidadãos de direito, a exigir os resultados efetivos dos serviços públicos (Melo, Alchieri, \& Araujo, 2012a). Salientando, entretanto, que a maioria dessas pesquisas de avaliação contemplam apenas os resultados dos programas (Belloni et al., 2003), de forma que são mais representativas as avaliações do PSF/ESF realizadas através da queixa de seus usuários, não sendo dedicada a devida atenção à avaliação sobre o perfil de seus profissionais e gestores (Martins, 2008; Melo, 2009; Melo et al., 2012a; Melo, Alchieri, \& Araujo, 2012b; 2013). Pretende-se no presente estudo, portanto, realizar uma avaliação da ESF através das crenças de seus profissionais, que são seus implementadores. Estes se tornam, assim, fonte preciosa de informação, pois, além de conhecerem a realidade do cotidiano da estratégia, são sujeitos que também possuem o poder de modificá-la, uma vez que esta é operacionalizada por suas mãos.

A maioria dos estudos constantes na literatura ainda é feito a partir das perspectivas dos sociólogos e economistas, havendo ainda pouca participação dos psicólogos em tal tarefa. Profissional que seria qualificado para essa prática, já que o mesmo é mais preparado profissionalmente para construção de instrumentos de avaliação de comportamento (Albuquerque, 1999). Defende-se, pois, que a escolha da metodologia, em especial do instrumento que será utilizado é uma decisão fundamental para a pesquisa de avaliação e é preocupação de diversos autores (Frekete, 2000; Furtado, 2001). Novaes (2000) também alerta para o fato de que as propostas e práticas de avaliação em saúde são importadas de outros países, onde há um maior desenvolvimento desta área, sendo necessária uma reinterpretação e ressignificação destas práticas, para que possam fazer sentido na realidade brasileira, devendo-se priorizar a construção de instrumentos de medidas próprios, adequados ao contexto da cultura em que vivem os participantes da pesquisa. Deste modo, alguns estudos já têm sido desenvolvidos no Brasil, sob o objetivo de criar tais ferramentas de avaliação do PSF/ESF, sob a perspectiva de seus profissionais. Oliveira (2010) criou um instrumento brasileiro, validada com amostra não probabilística de 220 profissionais em seis municípios do interior paraibano, com 38 itens dicotômicos, distribuídos em 5 fatores: condições de trabalho na $\operatorname{ESF}(\alpha=0,86)$, sistema de referência e contrarreferência $(\alpha=0,76)$, acessibilidade ao atendimento $(\alpha=0,59)$, compreensão da $\operatorname{ESF}(\alpha=0,65)$ e capacitação profissional $(\alpha=0,68)$. E Melo (2009) criou uma escala likert de 24 itens, com três fatores: recursos materiais $(\alpha=0,86)$, eficiência no atendimento $(\alpha=0,80)$ e infraestrutura física $(\alpha=0,73)$, validada na capital paraibana com amostra probabilística de 337 profissionais. Escalas com valores psicométricos muito bons, mas que podem ser melhorados, tanto nos índices de consistência interna, bem como em pontos de resposta mais fáceis de serem compreendidos para qualquer amostra de profissionais, independente do nível de escolarização.

Nesse sentido, a presente pesquisa abordou os profissionais da ESF sob o aspecto cognitivo, utilizando a teoria das crenças da Psicologia Social, partindo do suposto de Rokeach (1981) de que todas as pessoas possuem crenças, dentre elas, as crenças primitivas, adquiridas pela experiência, no contato direto com o objeto de crença. E teve ainda como suporte a teoria cognitivo-comportamental, que afirma que as cognições (eventos) estão baseadas em atividades ou suposições (esquemas) desenvolvidas a partir de experiências anteriores (Beck, 1982; Rangé, 2001); permitindo compreender que a partir das experiências cotidianas dentro das USF's os profissionais formam 
Melo, C. F., Alchieri, J. C., Araújo Neto, J. L. \& Melo, F. A. F. (2014). Desenvolvimento de Instrumentais para Avaliação da Estratégia Saúde da Família em Natal.

crenças sobre a ESF que afetam seu estado afetivo e padrão comportamental. Assim, os itens da escala criada não se basearam no conhecimento dos profissionais sobre aspectos teóricos da ESF, ou em idiossincrasias, nem na constatação direta de seu comportamento. Averiguou-se, sim, a percepção que os profissionais possuem sobre a realidade de funcionamento da ESF, através de crenças adquiridas no encontro direto com o objeto. Deste modo, a apreensão dessas crenças possibilitou a compreensão da realidade das condições de prestações de serviços de saúde para a comunidade, sob a óptica da Equipe de Saúde da Família, permitindo-nos, assim, identificar os aspectos cruciais que conduzem a atuação desses profissionais dentro da ESF.

\section{Objetivos}

A presente pesquisa objetivou criar e validar uma segunda versão da escala de Melo (2009), com nova nomenclatura: Escala Numérica de Avaliação da Estratégia Saúde da Família pelos profissionais, bem como avalia-la em Natal, Rio Grande do Norte. Para tanto, pretendeu-se: identificar as crenças dos profissionais das equipes multidisciplinares mínimas da ESF de Natal acerca do trabalho em ESF, no que se refere à Infraestrutura da USF (Fator 1); aos Recursos materiais (Fator 2); e sobre a Eficiência no atendimento, no que se refere ao Sistema de Referência e Contra referência e às capacitações (Fator 3); comparar as avaliações dos profissionais da EqSF em função do nível de escolaridade (nível médio e técnico $\mathrm{x}$ superior); comparar as avaliações dos profissionais da EqSF em função da categoria profissional (médico $x$ enfermeira $\mathrm{x}$ auxiliar de enfermagem...).

Assim, baseado na literatura de avaliação da ESF, se hipotetiza que:

1. H1 é possível aperfeiçoar psicometricamente as escalas para avaliar a ESF a partir das crenças de seus profissionais;

2. H2 os profissionais da ESF possuem crenças negativas sobre este serviço no que se refere ao fator geral de Infraestrutura da USF;

3. H3 os profissionais da EqSF possuem crenças positivas sobre a ESF no que se refere à disponibilidade de Recursos materiais na USF;

4. H4 os profissionais da ESF possuem crenças negativas sobre a Eficiência do atendimento;

5. H5 os profissionais do PSF com maior escolaridade (médico, enfermeira e dentista) possuem mais crenças negativas sobre a Infraestrutura da USF que os outros profissionais;

6. H6 os profissionais do PSF com menor escolaridade (médico, enfermeira e dentista) possuem mais crenças negativas sobre os Recursos materiais que os outros profissionais;

7. H7 os profissionais do PSF com maior escolaridade (médico, enfermeira e dentista) possuem mais crenças negativas sobre a eficiência no atendimen- to que os outros profissionais (ACS, ACD e auxiliar de enfermagem);

8. H8 os ACSs são os profissionais que pior avaliam a infraestrutura da USF;

9. H9 os ACSs são os profissionais que pior avaliam os recursos materiais disponíveis na USF;

10. H10 os médicos são os profissionais que pior avaliam as capacitações;

11. H11 os distritos mais pobres apresentam piores avaliações da ESF.

\section{Método}

\section{Delineamento}

Trata-se de uma pesquisa descritiva correlacional, aplicada, em que as variáveis antecedentes referem-se às condições de trabalho nas Unidades de Saúde da Família (USFs) e as variáveis consequentes referem-se à avaliação que os profissionais das equipes multidisciplinares mínimas da ESF fazem da estratégia.

\section{Participantes}

Utilizou-se uma amostragem probabilística composta por profissionais das equipes multidisciplinares mínimas da ESF (médico, dentistas, enfermeiros, auxiliares de enfermagem, auxiliares de consultório dentário - ACD e agentes comunitários de saúde - ACS) de Natal, selecionados através do randomizer a partir da listagem dos profissionais fornecida pela Secretaria Municipal de Saúde (SMS).

Contempla-se que existem, na cidade de Natal, 1187 profissionais ativos, distribuídos em 116 equipes, nas 37 USFs dos 5 Distritos Sanitários. Para uma população desse porte, com nível de confiança de 95\% e erro amostral de 5\%, fez-se necessário uma amostra mínima de 291 participantes.

Respeitando a diversidade da cidade e a heterogeneidade da quantidade de profissionais, distribuiu-se a amostra de forma estratificada por categoria profissional e por Distrito Sanitário. Após essa distribuição, teve-se ainda a cautela de manter um número mínimo de $30 \mathrm{su}$ jeitos por categoria profissional, valor mínimo por quota aconselhável para comparações estatísticas. Desta forma, a distribuição amostral aumentou para 475 profissionais, distribuídos em 25 unidades (Tabela 1).

Dá-se destaque ainda que, nessa amostragem probabilística, houve restrição de participantes devido a três motivos: (a) recusa do sorteado em participar da pesquisa; (b) o sorteado não trabalhar mais na USF ou os dados da Secretaria Municipal de Saúde (SMS) estarem desatualizados; (c) ausência do profissional sorteado (férias ou atestado). Desta forma, entre os ACSs, a substituição foi feita pelo profissional constado acima dele na lista do nome de todos os profissionais da cidade ou, em segunda opção, pelo nome abaixo. Já para as outras categorias de trabalhadores, que possuem apenas um profissional na equipe, foi realizado um novo sorteio. 
Psicologia: Reflexão e Crítica, 27(2), 219-227.

Tabela 1

Número de Sujeitos da População e (Participantes da Amostra) de Profissionais, Segundo Agrupamento Estratificado de Categoria Profissional e Distrito Sanitário

\begin{tabular}{lcccccc}
\hline \multicolumn{7}{c}{ População (amostra) } \\
$\begin{array}{l}\text { Categoria } \\
\text { profissional }\end{array}$ & $\begin{array}{c}\text { Distrito } \\
\text { Sanitário Sul }\end{array}$ & $\begin{array}{c}\text { Distrito Sanitário } \\
\text { Leste }\end{array}$ & $\begin{array}{c}\text { Distrito } \\
\text { Sanitário Oeste }\end{array}$ & $\begin{array}{c}\text { Distrito Sanitário } \\
\text { Norte I }\end{array}$ & $\begin{array}{c}\text { Distrito Sanitário } \\
\text { Norte II }\end{array}$ & $\begin{array}{c}\text { Total } \\
\text { Amostral }\end{array}$ \\
\hline Médico & $1(1)$ & $7(3)$ & $28(11)$ & $14(6)$ & $24(9)$ & 75 (30) \\
Dentistas & $2(1)$ & $9(3)$ & $30(12)$ & $28(11)$ & $32(13)$ & 101 (40) \\
ACD & $2(1)$ & $10(4)$ & $24(10)$ & $28(11)$ & $31(12)$ & 95 (38) \\
Enfermeiros & $3(1)$ & $12(5)$ & $39(15)$ & $27(11)$ & $32(13)$ & $113(\mathbf{4 5})$ \\
A.Enfermagem & $7(3)$ & $18(7)$ & $79(32)$ & $55(23)$ & $61(25)$ & 223 (90) \\
ACS & $18(7)$ & $47(20)$ & $206(82)$ & $147(59)$ & $161(64)$ & 580 (232) \\
Total & $33(\mathbf{1 0})$ & $121(\mathbf{2 7})$ & $440(\mathbf{1 2 7})$ & $319(\mathbf{7 7})$ & $363(\mathbf{8 8})$ & 1187 (475) \\
\hline
\end{tabular}

Nota. Em negrito, número de participantes da amostra.

\section{Instrumento}

Para a coleta de dados com os profissionais, foi desenvolvida e validada a segunda versão adaptada da escala de Melo (2009) e Melo, Alchieri, Araujo Neto e Melo (2014), descrita na introdução, com uma nova nomenclatura: Escala Numérica de Avaliação da Estratégia Saúde da Família pelos profissionais. O novo instrumento com 24 itens, agora formatados para uma escala de notas $(0 \mathrm{a}$ 10 ), investiga os mesmos fatores da escala original, porém com índices psicométricos superiores: (a) infraestrutura física (alpha $=0,84)$, com 6 itens - refere-se aos aspectos de infraestrutura física, instalações e à manutenção de materiais permanentes (instrumentos e equipamentos) nas USF; (b) recursos materiais (alpha $=0,85$ ), com 10 itens refletem as crenças que os profissionais da ESF têm sobre a disponibilidade de insumos para realização de seu trabalho; vacinas para o trabalho de imunização; e remédios e contraceptivos para distribuir aos usuários; (c) eficiência no atendimento (alpha $=0,82$ ), com 8 itens - reflete as crenças sobre o sistema de referência e contrarreferência e a capacitação profissional.

\section{Procedimentos Éticos e de Coleta de Dados}

Considerando-se os aspectos éticos referentes a pesquisas envolvendo seres humanos, inicialmente o estudo foi aprovado pela SMS e pela Comissão de Ética do Comitê de Ética em Pesquisa do Hospital Universitário Onofre Lopes - Rio Grande do Norte (CEP/HUOL - RN), sob o protocolo de $\mathrm{n}^{\circ} 0298$, em 09/07/2008. Após sorteada a amostra, foram, então, realizadas visitas às USFs, informando aos participantes os objetivos e procedimentos do estudo e solicitando, ainda, que eles assinassem um Termo de Consentimento Informado. O instrumento foi aplicado de forma individual, com garantia do anonimato da sua colaboração e da confidencialidade de suas respostas.

\section{Resultados}

Os resultados serão apresentados seguindo a ordem dos objetivos: a validação psicométrica da nova escala e a avaliação da ESF pelos profissionais em Natal.

\section{Escala Numérica de Avaliação da Estratégia Saúde da Família pelos seus Profissionais}

Antes de realizarem-se as análises sobre a avaliação que os profissionais que atuam nas Equipes de Saúde da Família (EqSF) fazem sobre a Estratégia Saúde da família (ESF), considerou-se a adequação de se efetuar uma análise fatorial com o conjunto de 24 itens, o que foi perfeitamente corroborado pelo valor do KMO $(0,88)$ e Teste de Esfericidade de Bartlett $\left(x^{2}=5171,23 ; p<0,05\right)$, sendo estes dados considerados ótimos, de acordo com Foster, Barkus e Yavorsky (2006). Destaca-se ainda que a análise de 475 sujeitos atendeu também o critério de Tabachnick e Fidel (2013) sobre o número mínimo da amostra ser cinco vezes maior que o número de itens, pois havia uma proporção de 19,79 pessoas/item.

Em seguida, foi realizada uma primeira análise fatorial, com uso do método "Componentes Principais" (Principal Components- PC), verificando-se que todos os itens possuem coeficientes com carga fatorial acima de 0,3 . Por fim, foi empregada a análise fatorial por meio do método do "Eixo Principal da Fatorial" (Principal Axis Factoring - PAF), com rotação varimax. Foram, então, encontrados 6 fatores com eigenvalues superiores a 1 , que explicavam $64,78 \%$ da variância total. Optando-se pelo arranjo com 3 fatores, por aglomerar melhor os itens no scree plot e ser mais adequado teoricamente.

Ao fim das análises, os três fatores mantidos, cujo suas definições constitutivas são as mesmas da escala original, apesar de valores psicométricos superiores, explicam 
Melo, C. F., Alchieri, J. C., Araújo Neto, J. L. \& Melo, F. A. F. (2014). Desenvolvimento de Instrumentais para Avaliação da Estratégia Saúde da Família em Natal.

$49,75 \%$ da variância total do construto estudado, possuem índice de consistência interna do Alpha de Cronbach excelente $(\alpha=0,91)$, ficando o instrumento final com 24 itens (sem perda de itens). Os resultados destas análises com os itens que compõem o instrumento final e suas cargas fatoriais podem ser observados na Tabela 2.

Tabela 2

Análise Fatorial da Escala Numérica de Avaliação da Estratégia Saúde da Família pelos seus Profissionais com Método da PAF

Itens

Fatores

Fator $1 \quad$ Fator 2 Fator 3

1 Que nota você atribui às salas de atendimento?

0,64

2 Que nota você atribui às condições de uso dos equipamentos?

0,57

3 Que nota você atribui à quantidade de instrumentos existentes?

0,69

4 Que nota você atribui às condições do prédio em que trabalha?

0,52

6 Que nota você atribui à quantidade de equipamentos existentes?

0,51

11 Que nota você atribui para os instrumentos que utiliza na USF (PSF)?

0,65

15.1 Que nota você atribui para a disponibilidade de luvas?

15.2 Que nota você atribui para a disponibilidade de gases?

15.3 Que nota você atribui para a disponibilidade de máscaras?

15.4 Que nota você atribui para a disponibilidade de amalgama?

15.5 Que nota você atribui para a disponibilidade de seringa?

0,76

15.6 Que nota você atribui para a disponibilidade de vacina?

15.7 Que nota você atribui para a disponibilidade de anestesia?

15.8 Que nota você atribui para a disponibilidade de remédios?

0,43

15.9 Que nota você atribui para a disponibilidade de camisinha?

0,71

15.10 Que nota você atribui para a disponibilidade de anticoncepcional?

0,60

5 Que nota você atribui à eficiência da central de marcação para encaminhamento?

7 Que nota você atribui à disponibilidade de capacitações oferecidas aos profissionais?

8 Que nota você atribui para as reuniões da equipe do PSF?

9 Que nota você atribui para a disponibilidade de treinamentos para o trabalho com a comunidade?

10 Que nota você atribui para a quantidade de vagas disponibilizadas para o encaminhamento?

12 Que nota você atribui para a contra referência?

13 Que nota você atribui para a qualidade das capacitações oferecidas pela Secretaria de Saúde?

14 Que nota você atribui para a referência dos atendimentos para níveis mais complexos?

O Fator 1, "Infraestrutura física", composto por 6 itens, com saturação mínima 0,51 [Item 6. Os equipamentos (cadeira do dentista, balança) da USF (ESF) existem em quantidade suficiente] e máxima 0,69 [Item 3. Os instrumentos (estetoscópio, prancheta, termômetro) da ESF (PSF) existem em quantidade suficiente]. Possui eigenvalue 1,61 , que explica $12,29 \%$ do índice de variância, com índice de consistência interna (Alpha de Cronbach, $\alpha$ ) igual a 0,84 . O fator é composto por itens que refletem a avaliação que os profissionais fazem sobre a infraestrutura das USFs no que se refere às instalações do prédio, e seus materiais permanentes (equipamentos e instrumentos), disponibilizados pelos gestores para otimização dos trabalhos dentro das unidades.

O Fator 2, "Recursos materiais", reuniu 10 itens, com saturação mínima 0,36 [Item 15.7 Disponibilidade de luvas] e máxima 0,73 [Item 15.5 Disponibilidade de vacina]. Este fator apresentou eigenvalue 8,01, que 
explica $16,08 \%$ do índice de variância, $\operatorname{com} \alpha=0,85$. Os itens que compõem este fator refletem as crenças que os profissionais da EqSF possuem sobre a disponibilidade dos recursos materiais nas Unidades de Saúde da Família (USFs).

O Fator 3, "Eficiência no atendimento", possui 8 itens, com saturação mínima 0,49 [Item 8 . Que nota você atribui para as reuniões da equipe do PSF] e máxima 0,65 [Item 10. Existem vagas suficientes para o encaminhamento dos pacientes para médicos especialistas e exames]. Este fator apresentou eigenvalue 2,32 , explicando $14,50 \%$ do índice de variância, $\operatorname{com} \alpha=0,82$.

$\mathrm{O}$ fator reflete a avaliação que os profissionais fazem sobre a eficiência no atendimento, considerando sua integração entre os seus três níveis (Atenção Básica, Secundária e Terciária) e qualidade de seus Recursos Humanos, dividindo-se em dois subfatores: "Sistema de referência e contra referência", com 4 itens, compreende a integração do SUS, avaliando a qualidade da integração da USF com os serviços dos demais níveis do sistema; e "Capacitação profissional", com 4 itens, englobando as crenças sobre a preparação inicial e continuada que os profissionais possuem para o trabalho em ESF. Observa-se, portanto, a confirmação da hipótese 1 , sendo oferecida uma escala aperfeiçoada com base nos pressupostos psicométricos, com fatores coerentes aos achados de Melo (2009) e Oliveira (2010), representativos da emergência dessa população.

\section{Análise Descritiva dos Fatores das Escalas de Avaliação da ESF pelos Profissionais}

Serão apresentados nesta seção os resultados encontrados nos fatores anteriormente descritos: (a) Recursos materiais; (b) Eficiência no atendimento; (c) Infraestrutura física. No primeiro momento serão apresentadas as medidas de tendência central para obtenção da nota geral do fator. Esta será considerada a partir da distribuição de um quartil: Péssimo (notas entre 0 a 2,5), Ruim (notas entre 2,5 a 5,0), Bom (notas entre 5,0 e 7,5) e Excelente (notas entre 7,5 e 10,0). Na segunda etapa, serão apresentadas comparações das avaliações de cada fator pelo nível de escolaridade dos profissionais (nível superior $\mathrm{x}$ nível técnico e médio), utilizando o teste $T$, e por categoria profissional e Distrito Sanitário, utilizando-se Anova.

Infraestrutura Física (Fator 1). Este fator é composto por itens que refletem o estado das Unidades de Saúde da Família (USF) no que se refere aos aspectos de infraestrutura física, instalações e manutenção de materiais permanentes (instrumentos e equipamentos), disponibilizados pelos gestores para a otimização do trabalho dos profissionais da ESF no oferecimento dos serviços de saúde aos seus usuários. A nota média geral atribuída a esse fator pelos seus profissionais foi de 4,37 $\pm 1,61 \mathrm{na}$ escala de 0 a 10 , admitindo-se, assim, uma "avaliação ruim", confirmando-se a hipótese 2.

E, ao comparar a avaliação realizada no fator "Infraestrutura física" por escolaridade dos profissionais, com aprovação da normalidade pelo teste Shapiro-Wilk $(D=0,99 ; p=0,7)$, verificou-se que os profissionais de nível superior $(4,71 \pm 1,46)$ e os profissionais de nível técnico e médio $(4,27 \pm 1,64)$ não apresentam diferenças significativas em suas avaliações deste fator $[t(475)=2,57$; $p>0,05]$, refutando-se a hipótese 5 , podendo-se inferir que independente do nível de escolaridade, os profissionais são prejudicados pelas condições físicas de trabalho.

Foram ainda realizadas comparações das médias do fator entre os seis grupos profissionais, observando-se que há diferença estatisticamente significativa $[F(5,47)=3,00$; $p<0,05]$. Enfatiza-se, através do post hoc Scheffé que os ACSs $(4,19 \pm 1,57)$ e os auxiliares de enfermagem $(4,17$ $\pm 1,79)$ apresentaram as menores médias de notas, confirmando a hipótese 8 ; e os $\operatorname{ACDs}(4,95 \pm 1,61)$ apresentaram as maiores notas (ver Tabela 3 ).

Tabela 3

Distribuição das Notas dos Fatores por Categoria Profissional

\begin{tabular}{lcccccc}
\hline & \multicolumn{5}{c}{ Média e DP } \\
\cline { 2 - 7 } & Médico & Enfermeira & $\begin{array}{c}\text { Auxiliar de } \\
\text { enfermagem }\end{array}$ & Dentista & ACD & ACS \\
\hline Fator 1: Infraestrutura física & $4,54 \pm 1,27$ & $4,76 \pm 1,50$ & $4,17 \pm 1,79$ & $4,77 \pm 1,57$ & $4,95 \pm 1,61$ & $1,19 \pm 1,57$ \\
Fator2: Recursos materiais & $4,54 \pm 1,20$ & $5,47 \pm 1,57$ & $5,17 \pm 1,78$ & $6,34 \pm 1,19$ & $6,34 \pm 1,1$ & $5,31 \pm 1,26$ \\
Fator 3: Eficiência no atendimento & $3,19 \pm 0,79$ & $4,02 \pm 1,24$ & $3,74 \pm 1,68$ & $4,06 \pm 1,24$ & $4,27 \pm 0,87$ & $4,12 \pm 0,33$ \\
\hline
\end{tabular}

Por fim, foram feitas comparações das médias do fator entre os 5 Distritos Sanitários (DS), observando-se que há diferença estatisticamente significativa $[F(4,47)=2,93 ; p$ $<0,05]$. Enfatiza-se, através do post hoc Scheffé que o DS Norte 2 apresentou a pior avaliação $(5,23 \pm 1,66)$ e o DS Leste apresentou a melhor avaliação $(5,86 \pm 1,22)$, em relação aos demais DS: Norte $1(5,67 \pm 1,48)$, DS Sul $(5,45 \pm 0,58)$ e DS Oeste $(5,27 \pm 1,36)$, confirmando a hipótese 11 .

Recursos materiais (Fator 2). Os itens que compõem este fator refletem as crenças que os profissionais da ESF têm sobre a disponibilidade de insumos para realização 
Melo, C. F., Alchieri, J. C., Araújo Neto, J. L. \& Melo, F. A. F. (2014). Desenvolvimento de Instrumentais para Avaliação da Estratégia Saúde da Família em Natal.

de seu trabalho (luvas, gases, máscaras, amálgama, seringas); vacinas para o trabalho de imunização; e remédios e contraceptivos para distribuir aos usuários. Obteve-se como nota geral de disponibilidade dos recursos materiais a mais alta dos três fatores, média de $5,42 \pm 1,47$, admitindo-se, assim, uma "avaliação boa", confirmando-se a hipótese 3.

Em seguida, foram realizadas as comparações da nota de 0 a 10 do fator por nível de escolaridade. Considerando a normalidade encontrada pelo teste Shapiro-Wilk, ( $D=0,99 ; p=0,06)$, utilizando o teste $T$, verificou-se que os profissionais de nível superior $(5,53 \pm 1,51)$ e os profissionais de nível técnico e médio $(5,38 \pm 1,46)$ não apresentam diferenças significativas em suas avaliações deste fator $[t(475)=0,94 ; p>0,05]$, refutando-se a hipótese 6 , onde se esperavam piores avaliações do nível médio e técnico, podendo-se, novamente, inferir que independente da escolaridade, os profissionais são prejudicados pelas condições materiais de trabalho.

Foram também realizadas comparações das médias do fator entre os 6 grupos profissionais, observando-se que há diferença estatisticamente significativa $[F(5,47)=9,90$; $P<0,05]$. Enfatizando, através do post hoc Scheffé que os ACSs $(5,31 \pm 1,26)$ foram os profissionais que apresentaram menor pontuação, confirmando hipótese 9 , provavelmente porque são a estes profissionais que mais são solicitados os recursos (camisinha, anticoncepcional e remédios) pela população e as maiores pontuações foram dos dentistas $(6,34 \pm 1,19)$ e ACDs $(6,34 \pm 1,41)$, provavelmente porque são os profissionais que menos tem contato com a farmácia, sala de vacinas (ver Tabela 3 ).

Por fim, foram feitas comparações das médias do fator entre os 5 Distritos Sanitários, observando-se que há diferença estatisticamente significativa $[F(4,47)=7,49 ; p<$ $0,05]$. Enfatiza-se, através do post hoc Scheffé que o DS Norte 2 destacou-se por ter a pior avaliação $(3,56 \pm 1,43)$ em relação aos demais DS: Norte $1(4,00 \pm 1,08)$, (DS Sul $4,87 \pm 1,52)$, DS Leste $(4,87 \pm 1,52)$ e DS Oeste $(4,11$ $\pm 1,36$ ), confirmando a hipótese 11 .

Eficiência no Atendimento (Fator 3). Este fator refere-se aos aspectos organizacionais das Unidades de Saúde da Família que abrangem o acesso da comunidade à ESF, verificando, segundo os profissionais, se o atendimento na ESF funciona ou não. Divide-se em dois subfatores: "Sistema de referência e contra referência", que abrange os encaminhamentos para outros níveis de complexidade; $\mathrm{e}$ "Capacitação profissional", que reflete as crenças dos profissionais da ESF sobre a existência, frequência e qualidade das capacitações profissionais e treinamentos oferecidos pelos gestores municipais de saúde aos profissionais da ESF, antes de começarem a trabalhar na ESF e durante o exercício de sua profissão. Obteve-se como nota geral da "Eficiência no atendimento", na escala de 0 a 10 , média $3,99$ ( $\pm 1,35)$, sendo o fator com média mais baixa, classificado como uma "avaliação ruim", confirmando-se a hipótese 4.
Nas comparações das pontuações da escala de 1 a 10 desse fator por escolaridade dos profissionais, considerando a normalidade encontrada pelo teste Shapiro-Wilk, ( $D=0,99 ; p=0,06)$, verificou-se que os profissionais de nível superior $(3,82 \pm 1,19)$ e profissionais de nível médio e técnico $(4,04 \pm 1,40)$, não apresentam diferenças significativas em suas avaliações deste fator $[t(475)=1,67$; $p<0,05]$, refutando-se a hipótese 7 .

Nas comparações das médias do fator entre os 6 grupos profissionais, observando-se que há diferença estatisticamente significativa $[F(5,46)=3,57 ; P<0,05]$. Enfatizando, através do post hoc Scheffé a diferenciação entre os ACSs $(4,12 \pm 0,33)$, com melhor pontuação, e os médicos $(3,19$ $\pm 0,79)$, profissionais com piores avaliações, podendo-se inferir que isso se deve ao fato de esses últimos serem os profissionais com maior rotatividade, e, consequentemente, os que participam de menos capacitações, confirmando hipótese 10, enquanto que os ACS são os profissionais que reconhecidamente são os que mais satisfazem as diretrizes de capacitação inicial e continuada. (ver Tabela 3 ).

Por fim, foram feitas comparações das médias do fator entre os 5 Distritos Sanitários, observando-se que há diferença estatisticamente significativa $[F(4,47)=14,57$; $p<0,05]$. Enfatiza-se, através do post hoc Scheffé que, desta vez, o DS Sul obteve pior avaliação $(2,06 \pm 0,71)$ em relação os demais DS: Norte $1(4,79 \pm 1,40)$, DS Norte $2(4,25 \pm 1,71)$, DS Leste $(5,21 \pm 1,25)$ e DS Oeste $(4,15 \pm 1,56)$, refutando-se a hipótese 11 , mostrando que apesar de esse ser o DS mais rico, com melhores índices de qualidade de vida, não funciona adequadamente a integração da ESF com os demais níveis do sistema e as capacitações profissionais.

\section{Discussão}

O objetivo principal deste estudo foi desenvolver e apresentar evidências de validade uma nova escala de avaliação da ESF no contexto brasileiro, levando em conta amostra específica de Natal. O objetivo foi alcançado, com dados estatisticamente excelente: com três fatores que explicam $49,75 \%$ da variância total do construto estudado e índice de Alpha de Cronbach de consistência interna geral classificada como excelente $(\alpha=0,91)$, apresentando-se, portanto, como um instrumento aprimorado, em relação aos existentes na literatura (Melo, 2009; Oliveira, 2010), atendendo às necessidades de instrumentos confiáveis e adaptados à realidade dos programas e estratégias brasileiras, como defende Belloni et al. (2003) e Novaes (2000). Algumas limitações podem, entretanto, ser levantadas nesta pesquisa, notadamente no que diz respeito aos participantes: embora tenha se tratado de amostra probabilística estratificada e representativa da população natalense, ela não é numericamente representativa da população brasileira. Não obstante, tais restrições devem ser ponderadas, uma vez que o propósito não foi generalizar os resultados, mas construir uma medida válida e confiável inicialmente 
com base num contexto local. Discutem-se a seguir os principais resultados.

Em relação à avaliação que os profissionais fazem da ESF, observou-se que a infraestrutura foi avaliada negativamente, com nota 4,37 $( \pm 1,61)$, dados que não corroboram com os resultados encontrados no estudo da primeira versão da escala (Melo, 2009) em João Pessoa (PB), onde esse é um fator normalmente bem avaliado, devido às novas unidades unificadas existentes nessa capital (Albuquerque \& Melo, 2010; Melo, 2009), bem diferentes das encontradas na realidade de Natal. Contempla-se, ainda, que esta não é uma exclusividade potiguar, pois, salvo algumas exceções, as más condições dos prédios das Unidades de Saúde da Família manifestam-se em diversas capitais, como mostrou a pesquisa, de caráter censitário, realizada pelo Ministério da Saúde, em todas as unidades da Federação (2004a, 2004b).

Os recursos materiais tiveram avaliação boa, com nota $5,42( \pm 1,47)$, fato que também surpreende, pois este é alvo de constante denuncias pelas pesquisas, devido à falta de luvas, gases, amálgamas, vacinas e remédios (Albuquerque \& Melo, 2010; Melo, 2009). Evidenciando-se que este é o único fator avaliado positivamente pelos profissionais da ESF em Natal.

E o último fator, eficiência no atendimento, foi avaliado negativamente $(3,99 \pm 1,35)$, evidenciando a falha no sistema de referência e falta de integração da ESF com o restante do sistema e escassez de capacitação profissional. Fato notório na literatura (Albuquerque \& Melo, 2010; Melo, 2009; Melo et al., 2012a).

\section{Considerações Finais}

Com base na discussão dos resultados, pôde-se perceber que, apesar de menos visíveis, os grandes entraves na realidade operacional do Sistema Único de Saúde e da Estratégia Saúde da Família (ESF), como sua porta de entrada na Atenção Básica, estão além das dificuldades apontadas pelos usuários. A partir da análise das crenças dos profissionais, foi possível descortinar as barreiras e entraves que dificultam a operacionalização de suas ações. Fato que só reforça a importância de contemplarem-se esses atores sociais nas pesquisas de avaliação da ESF, pois como são esses os verdadeiros implementadores das ações que são planejadas pelos governos federais, estaduais e municipais, são eles que se deparam com as incertezas e imprevistos inerentes a uma estratégia de ação e, consequentemente, são eles os sujeitos que reformulam em lócus suas adaptações necessárias, configurando-se, assim, como fontes preciosas de informação sobre a realidade operacional dos serviços de saúde ofertados à comunidade. Evidenciando, portanto, a necessidade de construção de ferramentas eficazes para esse tipo de tarefa, reforçando-se, em especial, a necessidade da participação dos psicólogos brasileiros, utilizando seu conhecimento sobre crenças grupais e psicometria, nas pesquisas de avaliação.
Sugere-se ainda a continuidade desse estudo, ampliando-o a outros estados brasileiros, para evidenciar aspectos da validade da escala às demais localidades e ampliação de seu poder de generalização, e assim, gerar feedback sobre o contexto dos serviços de saúde oferecidos pela ESF em todo território nacional.

Sabe-se ainda que a temática da saúde apresenta-se ampla e camuflada por princípios teóricos que não revelam sua realidade operacional falha. Compreende-se também que muitos tentam modificar o modelo de saúde brasileiro, o criticam, buscando a mudança das estruturas do SUS. Ao término deste trabalho, propõe-se, entretanto, que o problema não seja procurado nos papéis e nas formulações teóricas do modelo, e sim na operacionalização, no cotidiano do repasse de verbas, na capacitação dos profissionais, na infraestrutura dos locais de prestação de atendimento à comunidade. Trata-se de mitigar as barreiras que existem entre os dois extremos do sistema, aproximando a teoria e a prática. Trata-se de ter em mãos um sistema ideal, mas que necessita da operacionalização dessas formulações no cotidiano de suas ações. Contempla-se a necessidade de instigar a população a utilizar o serviço público, que não deve ser visto como algo apenas "para pobre" e, portanto, de baixa qualidade. É chegada a hora de lutar pela sua melhoria, incentivando a cultura de fiscalização dos gastos públicos e avaliação do retorno do investimento dado a esses serviços, transformados, de fato, em serviços de saúde eficientes e eficazes.

\section{Referências}

Albuquerque, F. J. B. de. (1999). Apontamentos para uma psicologia sócio-rural no Brasil. In Conselho Regional de Psicologia $-3^{\circ}$ Região, Anais do I Congresso Norte- Nordeste de Psicologia (Vol. 1, pp. 12-17). Salvador, BA: Conselho Regional de Psicologia $-3^{\circ}$ Região.

Albuquerque, F. J. B. (2002). Social psychology and rural life in Brazil. Psicologia: Teoria e Pesquisa, 18(1).

Albuquerque, F. J. B., \& Melo, C. F. (2010). Avaliação dos serviços públicos de saúde em duas capitais nordestinas do Brasil. Teoria e Pesquisa, 26, 323-330.

Albuquerque, F. J. B, Melo, C. F., Sousa Filho, F. E., \& Araujo Neto, J. L. (2011). Avaliação da estratégia saúde da família a partir das crenças dos profissionais. Estudos de Psicologia (PUCCAMP. Impresso), 28(3), 363-370.

Barroso, A. V. (2003). Mapeando a qualidade de vida em Natal. Natal, RN: Secretaria. Municipal de Planejamento e Gestão Estratégica.

Beck, A. T. (1982). Terapia cognitiva da depressão. Rio de Janeiro, RJ: Zahar.

Belloni, I., Magalhães, H., \& Sousa, L. C. (2003). Metodologia de avaliação em políticas públicas: Vol. 75. Nossa época (3. ed). São Paulo, SP: Cortez.

Foster, J. J., Barkus, E., \& Yavorsky, C. (2006). Understanding and using advanced statistics. Thousand Oaks, CA: Sage.

Frekete, M. C. (2000). Estudo da acessibilidade na avaliação dos serviços de saúde. Manuscrito não publicado.

Furtado, J. P. (2001). Um método construtivista para a avaliação em saúde. Ciência e Saúde Coletiva, 6(1), 165-181. 
Melo, C. F., Alchieri, J. C., Araújo Neto, J. L. \& Melo, F. A. F. (2014). Desenvolvimento de Instrumentais para Avaliação da Estratégia Saúde da Família em Natal.

Martins, C. (2008). Sistema Único de Saúde: Uma análise das crenças dos seus representantes em municípios rurais na Paraíba (Dissertação de mestrado, Universidade Federal da Paraíba, João Pessoa, PB, Brasil).

Melo, C. F. (2009). Avaliação da Estratégia Saúde da Família a partir das crenças de seus profissionais (Dissertação de mestrado, Universidade Federal da Paraíba, João Pessoa, PB, Brasil)

Melo, C. F., Alchieri, J. C., \& Araujo Neto, J. L. (2012a). Sistema Único de Saúde: Uma avaliação realizada em Natal, Rio Grande do Norte, Brasil. Psico-USF, 17(1), 323-330.

Melo, C. F., Alchieri, J. C., \& Araujo Neto, J. L. (2012b). Avaliação da Estratégia Saúde da Família a partir das crenças de seus gestores. Revista UFPE, 6(2), 274-278.

Melo, C. F., Alchieri, J. C., Araujo Neto, J. L., (2013). Evaluation of the technical and professional profile of managers of the brazilian Unified Health System (SUS). Revista de Enfermagem UFPE On Line, 7 (7), 4670-4680.

Melo, C. F., Alchieri, J. C., Araujo Neto, J. L., \& Melo, F. A. F. (2013). Avaliação da Estratégia Saúde da Família em Natal a partir das crenças dos seus colaboradores. Psico (PUCRS. Online), 44(4), 533-541.

Ministério da Saúde. (2001). Guia prático do Programa Saúde da Família. A saúde bucal faz parte da saúde da família? Brasília, DF: Autor.

Ministério da Saúde. (2004a). Atenção Básica e a Saúde da Família. Brasília, DF: Autor.

Ministério da Saúde. (2004b). Avaliação normativa do Programa Saúde da Família no Brasil: Monitoramento da implantação e funcionamento das equipes de saúde da família: 2001-2002. Brasília, DF: Autor.

Ministério da Saúde. (2006). Política Nacional de Atenção Básica: Vol. 4. Pactos pela saúde. Brasília, DF: Autor.

Novaes, H. M. D. (2000). Avaliação de programas, serviços e tecnologias em saúde. Revista de Saúde Pública, 34(5), 547-559.

Oliveira, S. F. (2010). Estratégia Saúde da Família: Avaliação das crenças dos profissionais que atuam em municípios rurais paraibanos (Tese de doutorado, Universidade Federal da Paraíba, João Pessoa, PB, Brasil).

Rangé, B. (2001). Psicoterapias cognitivo-comportamentais: Um diálogo com a psiquiatria. São Paulo, SP: Artmed.

Rokeach, M. (1981). Crenças, atitudes e valores. Rio de Janeiro, RJ: Interciência.

Tabachnick, B. G., \& Fidell, L. S. (2013). Using multivariate statistics $\left(6^{\text {th }}\right.$ ed.). Boston, MA: Allyn and Bacon. 